\title{
Summary and Recommendations
}

\author{
P. Kenneth Seidelmann \\ U. S. Naval Observatory \\ 3450 Massachusetts Ave., NW \\ Washington DC 20392-5420, USA \\ e-mail: pks@usno.navy.mil
}

\section{Summary}

The purpose of IAU Colloquium 180 was to prepare a series of resolutions regarding the specification of the International Celestial Reference System (ICRS) for consideration by Joint Discussion 2 at the XXIV IAU General Assembly. These resolutions provide definitions, standard algorithms, and parameters to link observable quantities with reference coordinates within the ICRS. Specifically recommended were definitions of the celestial intermediate pole and celestial ephemeris origin, an improved precession/nutation theory, and a new set of standard values for astronomical constants. These resolutions have far-reaching effects on positional and dynamical astronomy.

IAU Colloquium 180, attended by 96 participants, was organized with 32 invited speakers and 44 poster papers. Sessions were devoted to Celestial Reference Systems and Frames, Relativistic Considerations for High Precision Dynamical Astronomy, Precession and Nutation, Time and Constants, and Requirements for Improved Accuracy in Celestial Reference Systems. The colloquium also discussed plans for future observations of the defining elements of the celestial reference frames necessary to support present and planned high-precision astrometric programs. Time was scheduled to discuss these subjects and to prepare the appropriate resolutions. There were also opportunities to discuss the posters and for informal discussions concerning technical points about the proposed resolutions. Each resolution was given an initial presentation and thorough discussion. Drafting committees were appointed to revise the resolutions based on the discussion, and the revised resolutions were then discussed further. The process continued with revisions incorporated from the discussions until a final version of each resolution was adopted by a large majority of the attendees.

Resolutions with the following titles were adopted by the colloquium participants and forwarded to the IAU General Secretary for formal consideration and adoption at the XXIV IAU General Assembly in Manchester, England:

1. Maintenance and Establishment of Reference Frames and Systems

2. Hipparcos Celestial Reference Frame

3. Definition of Barycentric Celestial Reference System and Geocentric Celestial Reference System

4. Post-Newtonian Potential Coefficients 
5. Extended Relativistic Framework for Time Transformations and Realization of Coordinate Times in the Solar System

6. IAU 2000 Precession-Nutation Model

7. Definition of Celestial Intermediate Pole

8. Definition and Use of Celestial and Terrestrial Ephemeris Origin

9. Re-definition of Terrestrial Time TT

10. Coordinated Universal Time

\section{Resolutions}

Resolution 1. Maintenance and Establishment of Reference Frames and Systems

IAU Colloquium 180 "Towards Models and Constants for Sub-Microarcsecond Astrometry"

$\underline{\text { Noting }}$

1. that resolution B2 of the XXIIIrd General Assembly specifies that "the fundamental reference frame shall be the International Celestial Reference Frame (ICRF) constructed by the IAU Working Group on Reference Frames,"

2. that Resolution B2 of the XXIIIrd General Assembly specifies "That the Hipparcos Catalogue shall be the primary realization of the ICRS at optical wavelengths," and

3. the need for accurate definition of reference systems brought about by unprecedented precision, and

\section{$\underline{\text { Recognizing }}$}

1. the importance of continuing operational observations made with Very Long Baseline Interferometry (VLBI) to maintain the ICRF,

2. the importance of VLBI observations to the operational determination of the parameters needed to specify the time-variable transformation between the International Celestial and Terrestrial Reference Frames,

3. the progressive shift between the Hipparcos frame and the ICRF, and

4. the need to maintain the optical realization as close as possible to the ICRF 


\section{$\underline{\text { Recommends }}$}

1. that IAU Division I maintain the Working Group on Celestial Reference Systems formed from Division I members to consult with the International Earth Rotation Service (IERS) regarding the maintenance of the ICRS,

2. that the IAU recognize the International VLBI Service for Geodesy and Astrometry (IVS) as an IAU Service Organization,

3. that an official representative of the IVS be invited to participate in the IAU Working Group on Celestial Reference Systems,

4. that the IAU continue to provide an official representative to the IVS Directing Board,

5. that the astrometric and geodetic VLBI observing programs consider the requirements for maintenance of the ICRF and linking to the Hipparcos optical frame in the selection of sources to be observed (with emphasis on the Southern Hemisphere), design of observing networks, and the distribution of data, and

6. that the scientific community continue with high priority ground and space-based observations for the maintenance of the optical Hipparcos frame, frames at other wavelengths and for the links of the frames to the ICRF.

\section{Resolution 2. Hipparcos Celestial Reference Frame}

IAU Colloquium 180 "Towards Models and Constants for Sub-Microarcsecond Astrometry"

$\underline{\text { Noting }}$

1. that Resolution B2 of the XXIIIrd General Assembly specifies, "That the Hipparcos Catalogue shall be the primary realization of the ICRS at optical wavelengths,"

2. the need for this realization to be of the highest precision,

3. that the proper motions of many of the Hipparcos stars known, or suspected, to be multiple are adversely affected by uncorrected orbital motion,

4. the extensive use of the Hipparcos Catalogue as reference for the ICRS in works of densification and extension to fainter stars,

5. the need to avoid confusion between the ICRF and the Hipparcos frame, and 
6. the progressive shift between the Hipparcos frame and the ICRF,

\section{$\underline{\text { Recommends }}$}

1. that Resolution B2 of the XXIIIrd IAU General Assembly be amended by excluding from the optical realization of the ICRS all stars flagged $\mathrm{C}, \mathrm{G}$, $\mathrm{O}, \mathrm{V}$ and $\mathrm{X}$ in the Hipparcos Catalogue, and

2. that this modified Hipparcos frame be labeled HCRF: Hipparcos Celestial Reference Frame

\section{Resolution 3. Definition of Barycentric Celestial Reference System and Geocentric Celestial Reference System}

IAU Colloquium 180 "Towards Models and Constants for Sub-Microarcsecond Astrometry"

\section{Considering}

1. that the IAU Resolution A4 (1991) has defined a system of space-time coordinates for the solar system (now called the Barycentric Celestial Reference System, BCRS) and one for the Earth (now called the Geocentric Celestial Reference System, GCRS), within the framework of General Relativity,

2. the desire to write the metric tensors both in the BCRS and in the GCRS in a compact and self-consistent form

3. the fact that tremendous work in General Relativity has been done using the harmonic gauge, which was found to be a useful and simplifying gauge for many kinds of applications,

\section{$\underline{\text { Recommends }}$}

1. to choose harmonic coordinates both for the barycentric and for the geocentric reference systems.

2. to write the time-time component and the space-space component of the barycentric metric $g_{\mu \nu}$ with barycentric coordinates $(t, \mathbf{x})(t=\mathrm{TCB})$ with one single scalar potential $w(t, \mathbf{x})$ that generalizes the Newtonian potential, and the space-time component with a vector potential $w^{i}(t, x)$. As a boundary condition it is assumed that these two potentials vanish far from the solar system.

Explicitly,

$$
g_{00}=-1+\frac{2 w}{c^{2}}-\frac{2 w^{2}}{c^{4}}
$$




$$
\begin{gathered}
g_{0 i}=-\frac{4}{c^{3}} w^{i} \\
g_{i j}=\delta_{i j}\left(1+\frac{2}{c^{2}} w\right),
\end{gathered}
$$

with

$$
\begin{gathered}
w(t, \mathbf{x})=G \int d^{3} x^{\prime} \frac{\sigma\left(t \mathbf{x}^{\prime}\right)}{\left|\mathbf{x}-\mathbf{x}^{\prime}\right|}+\frac{1}{2 c^{2}} G \frac{\partial^{2}}{\partial t^{2}} \int d^{3} x^{\prime} \sigma\left(t, \mathbf{x}^{\prime}\right)\left|\mathbf{x}-\mathbf{x}^{\prime}\right| \\
w^{i}(t, \mathbf{x})=G \int d^{3} x^{\prime} \frac{\sigma^{i}\left(t, \mathbf{x}^{\prime}\right)}{\left|\mathbf{x}-\mathbf{x}^{\prime}\right|}
\end{gathered}
$$

Here, $\sigma$ and $\sigma_{i}$ are the gravitational mass and current densities, respectively.

3. to write the geocentric metric tensor $G_{\alpha \beta}$ with geocentric coordinates $(T, \mathbf{X})(T=$ TCG) in the same form as the barycentric one but with potentials $W(T, \mathbf{X})$ and $W^{a}(T, \mathbf{X})$. These geocentric potentials should be split into two parts: potentials $W_{E}$ and $W_{E}^{a}$ arising from the gravitational action of the Earth and external parts $W_{\text {ext }}$ and $W_{\text {ext }}^{a}$ due to tidal and inertial effects. The external parts of the metric potentials are assumed to vanish at the geocenter and admit an expansion into positive powers of $\mathbf{X}$. Explicitly,

$$
\begin{gathered}
G_{00}=-1+\frac{2 W}{c^{2}}-\frac{2 W^{2}}{c^{4}} \\
G_{0 a}=-\frac{4}{c^{3}} W^{a} \\
G_{a b}=\delta_{a b}\left(1+\frac{2}{c^{2}} W\right) .
\end{gathered}
$$

The potentials $W$ and $W^{a}$ should be split according to

$$
\begin{gathered}
W(T, \mathbf{X})=W_{E}(T, \mathbf{X})+W_{\text {ext }}(T, \mathbf{X}), \\
W^{a}(T, \mathbf{X})=W_{E}^{a}(T, \mathbf{X})+W_{\text {ext }}^{a}(T, \mathbf{X}) .
\end{gathered}
$$

The Earth's potentials $W_{E}$ and $W_{E}^{a}$ are defined in the same way as $w_{E}$ and $w_{E}^{i}$ but with quantities calculated in the GCRS.

4. if required by accuracy, to use the full post-Newtonian coordinate transformation between the BCRS and the GCRS as induced by the form of the corresponding metric tensors. Explicitly, for the kinematically nonrotating GCRS ( $T=\mathrm{TCG}, t=\mathrm{TCB}, r_{E}^{i} \equiv x^{i}-x_{E}^{i}(t)$ and a summation from 1 to 3 over equal indices is implied), 


$$
\begin{gathered}
T=t-\frac{1}{c^{2}}\left[A(t)+v_{E}^{i} r_{E}^{i}\right] \\
+\frac{1}{c^{4}}\left[B(t)+B^{i}(t) r_{E}^{i}+B^{i j}(t) r_{E}^{i} r_{E}^{j}+C(t, \mathbf{x})\right]+\mathcal{O}\left(c^{-5}\right) \\
X^{a}=\delta_{a i}\left[r_{E}^{i}+\frac{1}{c^{2}}\left(\frac{1}{2} v_{E}^{i} v_{E}^{j} r_{E}^{j}+w_{\mathrm{ext}}\left(\mathbf{x}_{E}\right) r_{E}^{i}+r_{E}^{i} a_{E}^{j} r_{E}^{j}-\frac{1}{2} a_{E}^{i} r_{E}^{2}\right)\right]+\mathcal{O}\left(c^{-4}\right)
\end{gathered}
$$

where

$$
\begin{gathered}
\frac{d}{d t} A(t)=\frac{1}{2} v_{E}^{2}+w_{\mathrm{ext}}\left(\mathbf{x}_{E}\right) \\
\frac{d}{d t} B(t)=-\frac{1}{8} v_{E}^{4}-\frac{3}{2} v_{E}^{2} w_{\mathrm{ext}}\left(\mathbf{x}_{E}\right)+4 v_{E}^{i} w_{\mathrm{ext}}^{i}\left(\mathbf{x}_{E}\right)+\frac{1}{2} w_{\mathrm{ext}}^{2}\left(\mathbf{x}_{E}\right) \\
B^{i}(t)=-\frac{1}{2} v_{E}^{2} v_{E}^{i}+4 w_{\mathrm{ext}}^{i}\left(\mathbf{x}_{E}\right)-3 v_{E}^{i} w_{\mathrm{ext}}\left(\mathbf{x}_{E}\right) \\
B^{i j}(t)=-v_{E}^{i} \delta_{a j} Q^{a}+2 \frac{\partial}{\partial x^{j}} w_{\mathrm{ext}}^{i}\left(\mathbf{x}_{E}\right)-v_{E}^{i} \frac{\partial}{\partial x^{j}} w_{\mathrm{ext}}\left(\mathbf{x}_{E}\right)+\frac{1}{2} \delta^{i j} \dot{w}_{\mathrm{ext}}\left(\mathbf{x}_{E}\right) \\
C(t, \mathbf{x})=-\frac{1}{10} r_{E}^{2}\left(\dot{a}_{E}^{i} r_{E}^{i}\right) .
\end{gathered}
$$

Here $x_{E}^{i}, v_{E}^{i}$, and $a_{E}^{i}$ are the barycentric position, velocity and acceleration vectors of the Earth, the dot stands for the total derivative with respect to $t$, and

$$
Q^{a}=\delta_{a i}\left[\frac{\partial}{\partial x_{i}} w_{\mathrm{ext}}\left(\mathbf{x}_{E}\right)-a_{E}^{i}\right]
$$

The external potentials, $w_{\text {ext }}$ and $w_{\text {ext }}^{i}$, are given by

$$
w_{\mathrm{ext}}=\sum_{A \neq E} w_{A} ; \quad w_{\mathrm{ext}}^{i}=\sum_{A \neq E} w_{A}^{i},
$$

where $E$ stands for the Earth and $w_{A}$ and $w_{A}^{i}$ are determined by the expressions for $w$ and $w^{i}$ with integrals taken over body $A$ only. 
Note It is to be understood that these expressions for $w$ and $w^{i}$ give $g_{00}$ correct up to $\mathcal{O}\left(c^{-5}\right), g_{0 i}$ up to $\mathcal{O}\left(c^{-5}\right)$, and $g_{i j}$ up to $\mathcal{O}\left(c^{-4}\right)$. The densities $\sigma$ and $\sigma^{i}$ are determined by the components of the energy momentum tensor of the matter composing the solar system bodies as given in the accompanying literature. Accuracies for $G_{\alpha \beta}$ in terms of $c^{-n}$ correspond to those of $g_{\mu \nu}$.

The external potentials $W_{\text {ext }}$ and $W_{\text {ext }}^{a}$ can be written in the form

$$
\begin{aligned}
& W_{\text {ext }}=W_{\text {tidal }}+W_{\text {iner }}, \\
& W_{\text {ext }}^{a}=W_{\text {tidal }}^{a}+W_{\text {iner }}^{a} .
\end{aligned}
$$

$W_{\text {tidal }}$ generalizes the Newtonian expression for the tidal potential. Post-Newtonian expressions for $W_{\text {tidal }}$ and $W_{\text {tidal }}^{a}$ can be found in the accompanying literature. The potentials $W_{\text {iner }}, W_{\text {iner }}^{a}$ are inertial contributions that are linear in $X^{a}$. The former is determined mainly by the coupling of the Earth's nonsphericity to the external potential. In the kinematically non-rotating Geocentric Celestial Reference System, $W_{\text {iner }}^{a}$ describes the Coriolis force induced mainly by geodetic precession.

Finally, the local gravitational potentials $W_{E}$ and $W_{E}^{a}$ of the Earth are related to the barycentric gravitational potentials $w_{E}$ and $w_{E}^{i}$ by

$$
\begin{aligned}
& W_{E}(T, \mathbf{X})=w_{E}(t, \mathbf{x})\left(1+\frac{2}{c^{2}} v_{E}^{2}\right)-\frac{4}{c^{2}} v_{E}^{i} w_{E}^{i}(t, \mathbf{x})+\mathcal{O}\left(c^{-4}\right) \\
& W_{E}^{a}(T, \mathbf{X})=\delta_{a i}\left(w_{E}^{i}(t, \mathbf{x})-v_{E}^{i} w_{E}(t, \mathbf{x})\right)+\mathcal{O}\left(c^{-2}\right)
\end{aligned}
$$

\section{References}

Brumberg, V.A., Kopeikin, S.M., 1988, Nuovo Cimento, B103, 63.

Brumberg, V.A., 1991, Essential Relativistic Celestial Mechanics, Hilger, Bristol. Damour, T., Soffel, M., Xu, C., Phys. Rev. D, 43, 3273 (1991); 45, 1017 (1992); 47, 3124 (1993); 49, 618 (1994).

Klioner, S. A., Voinov, A.V., 1993, Phys Rev. D, 48, 1451.

Kopeikin, S.M., 1989, Celest. Mech., 44, 87.

\section{Resolution 4. Post-Newtonian Potential Coefficients}

IAU Colloquium 180 "Towards Models and Constants for Sub-Microarcsecond Astrometry"

\section{Considering}

1. that for many applications in the fields of celestial mechanics and astrometry a suitable parametrization of the metric potentials (or multipole moments) outside the massive solar-system bodies in form of expansions in terms of potential coefficients are extremely useful, and 
2. that physically meaningful post-Newtonian potential coefficients can be derived from the literature,

\section{$\underline{\text { Recommends }}$}

1. to expand the post-Newtonian Earth's potential $W_{E}$ in the Geocentric Celestial Reference System (GCRS) outside the Earth in the form

$W_{\mathrm{E}}(T, \mathbf{X})=\frac{G M_{\mathrm{E}}}{R}\left[1+\sum_{l=2}^{\infty} \sum_{m=0}^{+l}\left(\frac{R_{\mathrm{E}}}{R}\right)^{l} P_{l m}(\cos \theta)\left(C_{l m}^{E}(T) \cos m \phi+S_{l m}^{E}(T) \sin m \phi\right)\right]$

Here $C_{l m}^{E}$ and $S_{l m}^{E}$ are, to sufficient accuracy, equivalent to the postNewtonian multipole moments introduced in (Damour et al., 1991, Phys. Rev. $D, 43,3273) . \theta$ and $\phi$ are the polar angles corresponding to the spatial coordinates $X^{a}$ of the GCRS and $R=|\mathbf{X}|$, and

2. to express the vector potential $W_{E}^{a}$ outside the Earth, leading to the wellknown Lense-Thirring effect, in terms of the Earth's total angular momentum vector $\mathbf{S}_{E}$ in the form

$$
W_{E}^{a}(T, \mathbf{X})=-\frac{G}{2} \frac{\left(\mathbf{X} \times \mathbf{S}_{\mathrm{E}}\right)^{a}}{R^{3}}
$$

Resolution 5. Extended Relativistic Framework for Time Transformations and Realization of Coordinate Times in the Solar System

IAU Colloquium 180 "Towards Models and Constants for Sub-Microarcsecond Astrometry"

Considering

1. that the IAU Resolution A4 (1991) has defined systems of space-time coordinates for the solar system (Barycentric Reference System) and for the Earth (Geocentric Reference System), within the framework of General Relativity,

2. that Resolution 3 has renamed these systems the Barycentric Celestial Reference System (BCRS) and the Geocentric Celestial Reference System, respectively, and has specified a general framework for expressing their metric tensor and defining coordinate transformations at the first postNewtonian level,

3. that, based on the anticipated performance of atomic clocks, future time and frequency measurements will require practical application of this framework in the BCRS, 
4. that theoretical work requiring such expansions has already been performed,

Recommends that for applications that concern time transformations and realization of coordinate times within the solar system, Resolution 3 be applied as follows:

1. the metric tensor be expressed as

$$
\begin{gathered}
g_{00}=-\left(1-\frac{1}{c^{2}}\left(w_{0}(t, \mathbf{x})+w_{L}(t, \mathbf{x})\right)+\frac{2}{c^{4}}\left(w_{0}^{2}(t, \mathbf{x})+\Delta(t, \mathbf{x})\right)\right), \\
g_{0 i}=-\frac{4}{c^{3}} w^{i}(t, \mathbf{x}), \\
g_{i j}=\left(1+\frac{2 w_{0}(t, \mathbf{x})}{c^{2}}\right) \delta_{i j},
\end{gathered}
$$

where $(t \equiv$ Barycentric Coordinate Time (TCB), $\mathbf{x})$ are the barycentric coordinates, $w_{0}=G \sum_{A} \frac{M_{A}}{r_{A}}$, with the summation carried out over all solar system bodies $A, \mathbf{r}_{A}=\mathbf{x}-\mathbf{x}_{A}, x_{A}$ are the coordinates of the center of mass of body $A, r_{A}=\left|\mathbf{r}_{A}\right|$, and where $w_{L}$ contains the expansion in terms of multipole moments [see their definition in Resolution 4] required for each body. The vector potential $w^{i}(t, x)=\sum_{A}(t, x)$, and the function $\Delta(t, \mathbf{x})=\sum_{A} \Delta_{A}(t, \mathbf{x})$ are given in note 2.

2. the relation between TCB and Geocentric Coordinate Time (TCG) can be expressed to sufficient accuracy by

$$
\begin{gathered}
T C B-T C G=c^{-2}\left[\int_{t_{0}}^{t}\left(\frac{v_{E}^{2}}{2}+w_{0 \mathrm{ext}}\left(x_{E}\right) d t\right)+v_{E}^{i} r_{E}^{i}\right] \\
-c^{-4}\left[\int_{t_{0}}^{t}\left(-\frac{1}{8} v_{e}^{4}-\frac{3}{2} v_{E}^{2} w_{0 \mathrm{ext}}\left(x_{E}\right)+4 v_{E}^{i} w_{\mathrm{ext}}^{i}\left(x_{E}\right)+\frac{1}{2} w_{0 \mathrm{ext}}^{2}\left(x_{E}\right)\right) d t-\left(3 w_{0 \mathrm{ext}}\left(x_{E}\right)+\frac{v_{E}^{2}}{2}\right) v_{E}^{i}\right.
\end{gathered}
$$

where $v_{E}$ is the barycentric velocity of the Earth and where the index ext refers to summation over all bodies except the Earth.

\section{$\underline{\text { Notes }}$}

1. This formulation will provide an uncertainty not larger than $5 \times 10^{-18}$ in rate and, for quasi-periodic terms, not larger than $5 \times 10^{-18}$ in rate amplitude and $0.2 \mathrm{ps}$ in phase amplitude, for locations farther than a few solar radii from the Sun. The same uncertainty also applies to the transformation between TCB and TCG for locations within $50000 \mathrm{~km}$ of the Earth. Uncertainties in the values of astronomical quantities may induce larger errors in the formulas. 
2. Within the above mentioned uncertainties, it is sufficient to express the vector potential $w_{A}^{i}(t, \mathbf{x})$ of body $A$ as

$$
w_{A}^{i}(t, x)=G\left[\frac{-\left(r_{A} \times S_{A}\right)^{i}}{2 r_{A}^{3}}+\frac{M_{A} v_{A}^{i}}{r_{A}}\right]
$$

where $\mathrm{S}_{A}$ is the total angular momentum of body $A$ and $v_{A}^{i}$ is the barycentric coordinate velocity of body $A$. As for the function $\Delta_{A}(t, \mathbf{x})$ it is sufficient to express it as

$$
\begin{gathered}
\Delta_{A}(t, x)=\frac{G M_{A}}{r_{A}}\left[-2 v_{A}^{2}+\sum_{B \neq A} \frac{G M_{B}}{r_{B A}}+\frac{1}{2}\left(\frac{\left(r_{A}^{k} v_{A}^{k}\right)^{2}}{r_{A}^{2}}+r_{A}^{k} a_{A}^{k}\right)\right] \\
-\frac{2 G v_{A}^{k}\left(r_{A} \times S_{A}\right)^{k}}{r_{A}^{3}}
\end{gathered}
$$

where $r_{B A}=\left|\mathbf{X}_{B}-\mathbf{x}_{A}\right|$ and $a_{A}^{k}$ is the barycentric coordinate acceleration of body $A$. In these formulas, the terms in $\mathbf{S}_{A}$ are needed only for Jupiter $\left(S \approx 6.9 \times 10^{38} \mathrm{~m}^{2} \mathrm{~s}^{-1} \mathrm{~kg}\right)$ and Saturn $\left(S \approx 1.4 \times 10^{38} \mathrm{~m}^{2} \mathrm{~s}^{-1} \mathrm{~kg}\right)$, in the immediate vicinity of these planets.

3. Because the present Recommendation provides an extension of the IAU 1991 recommendations valid at the full first post-Newtonian level, the constants $L_{C}$ and $L_{B}$ that were introduced in the IAU 1991 recommendations should be defined as $\langle\mathrm{TCG} / \mathrm{TCB}\rangle=1-L_{C}$ and $\langle\mathrm{TT} / \mathrm{TCB}\rangle=$ $1-L_{B}$, where $<>$ refers to a sufficiently long average. The most recent estimate of $L_{C}$ is (Irwin and Fukushima, 1999)

$$
L_{C}=1.48082686741 \times 10^{-8} \pm 2 \times 10^{-17} .
$$

From Resolution 9 one infers $L_{B}=1.55051976772 \times 10^{-8} \pm 2 \times 10^{-17}$ by using the relation $1-L_{B}=\left(1-L_{C}\right)\left(1-L_{G}\right)$.

Because no unambiguous definition may be provided for $L_{B}$ and $L_{C}$, these constants should not be used in formulating time transformations when it would require knowing their value with an uncertainty of order $1 \times 10^{-16}$ or less.

4. If TCB-TCG is computed using planetary ephemerides which are expressed in terms of a time argument (noted $T_{e p h}$ ) which is close to Barycentric Dynamical Time (TDB), rather than in terms of TCB, the first integral in (1) may be computed as

$$
\int_{t_{0}}^{t}\left(\frac{v_{e}^{2}}{2}+w_{0 \mathrm{ext}}\left(x_{E}\right)\right) d t=\left[\int_{T_{\text {eph }}}^{T_{\text {eph }}}\left(\frac{v_{E}^{2}}{2}+w_{0 \mathrm{ext}}\left(x_{E}\right)\right) d t\right] /\left(1-L_{B}\right) .
$$


Resolution 6. IAU 2000 Precession-Nutation Model

IAU Colloquium 180 "Towards Models and Constants for Sub-Microarcsecond Astrometry"

$\underline{\text { Recognizing }}$

1. that the International Astronomical Union and the International Union of Geodesy and Geophysics Working Group (IAU-IUGG WG) on 'Non-rigid Earth Nutation Theory' has met its goals by

(a) establishing new high precision rigid-Earth nutation series, such as (1) SMART97 of Bretagnon et al., 1998, A\&A, 329, 329-338; (2) REN2000 of Souchay et al., 1999, A\&A S Supl. Ser, 135, 111-131; (3) RDAN97 of Roosbeek and Dehant 1999, Celest. Mech., 70, 215-253;

(b) completing the comparison of new nonrigid-Earth transfer functions for an Earth initially in non-hydrostatic equilibrium, incorporating mantle anelasticity and a Free Core Nutation period in agreement with observations,

(c) taking note that numerical integration models are not yet ready to incorporate dissipation in the core, and

(d) taking note of the effects of other geophysical and astronomical phenomena that must be modeled, such as ocean and atmospheric tides, that need further development;

2. that, as instructed by IAU Recommendation C1 in 1994, the International Earth Rotation Service (IERS) has published in the IERS Conventions (2000) a precession-nutation model that matches the observations with a weighted $\mathrm{rms}$ of 0.2 milliarcsecond (mas);

3. that semi-analytical geophysical theories of forced nutation are available which incorporate some or all of the following: anelasticity and electromagnetic couplings at the core-mantle and inner core-outer core boundaries, annual atmospheric tide, geodesic nutation, and ocean tide effects;

4. that ocean tide corrections are necessary at all nutation frequencies; and

5. that empirical models based on a resonance formula without further corrections do also exist;

Endorses the conclusions of the IAU-IUGG WG on Non-rigid Earth Nutation Theory published in Celestial Mechanics and Dynamical Astronomy (Dehant et al., 1999, 72(4), 245-310) and the recent comparisons between the various possibilities, and

Recommends that, beginning on 1 January 2003, the scientific community, in replacement of the IAU 1976 Precession Model and IAU 1980 Theory of Nutation, use the precession-nutation model IAU $2000 \mathrm{~A}$ for those who need a model at the 
0.2 mas level, or its shorter version IAU 2000B for those who need a model only at the 1 mas level, together with their associated precession and obliquity rates, and their associated celestial pole offsets, as published in the IERS Conventions 2000 , and

\section{Encourages}

1. further theoretical developments of the transfer functions including dissipation in the core and at its boundaries,

2. the continuation of theoretical developments of nonrigid-Earth nutation series for increasing the coherence between the different approaches and for incorporating the mutual influences of UT1 variations and nutations,

3. the continuation of VLBI observations for increasing the accuracy of the nutation series and the nutation model, and getting the observation of unpredictable free core nutation, and

4. the development of new expressions for precession consistent with the IAU $2000 \mathrm{~A}$ model.

\section{Resolution 7. Definition of Celestial Intermediate Pole}

IAU Colloquium 180 "Towards Models and Constants for Sub-Microarcsecond Astrometry"

Noting the need for accurate definition of reference systems brought about by unprecedented observational precision, and

\section{$\underline{\text { Recognizing }}$}

1. the need to specify an axis with respect to which the Earth's angle of rotation is defined,

2. that the Celestial Ephemeris Pole does not take account of diurnal and higher frequency variations in the Earth's orientation,

\section{$\underline{\text { Recommends }}$}

1. that the Celestial Intermediate Pole (CIP) be the pole, the motion of which is specified in the Geocentric Celestial Reference System (see Resolution 3 ) by motion of the Tisserand mean axis of the Earth with periods greater than two days,

2. that the direction of the CIP at J2000.0 be offset from the direction of the pole of the GCRS in a manner consistent with the IAU $2000 \mathrm{~A}$ (see Resolution 6) precession-nutation model, 
3. that the motion of the CIP in the Geocentric Celestial Reference System be realized by the IAU $2000 \mathrm{~A}$ model for precession and forced nutation for periods greater than two days plus additional time-dependent corrections provided by the International Earth Rotation Service (IERS) through appropriate astro-geodetic observations,

4. that the motion of the CIP in the International Terrestrial Reference System be provided by the International Earth Rotation Service through appropriate astro-geodetic observations and models including high-frequency variations,

5. that for highest precision, corrections to the models for the motion of the CIP in the International Terrestrial Reference System may be estimated using procedures specified by the IERS, and

6. that implementation of the CIP be on 1 January 2003.

Note The forced nutations with periods less than two days are included in the model for the motion of the CIP in the ITRS. The Tisserand mean axis of the Earth corresponds to the mean surface geographic axis, quoted B axis, in Seidelmann (1982). As a consequence of this resolution, the Celestial Ephemeris Pole is no longer necessary.

\section{Resolution 8. Definition and use of Celestial and Terrestrial Ephemeris Origin}

IAU Colloquium 180 "Towards Models and Constants for Sub-Microarcsecond Astrometry"

$\underline{\text { Recognizing }}$

1. the need for reference system definitions suitable for modern realizations of the conventional reference systems and consistent with observational precision,

2. the need for a rigorous definition of sidereal rotation of the Earth,

3. the desirability of describing the rotation of the Earth independently from its orbital motion, and

Noting that the use of the "non-rotating origin" (Guinot, 1979) on the moving equator fulfills the above conditions and allows for a definition of UT1 which is insensitive to changes in models for precession and nutation at the microarcsecond level 


\section{$\underline{\text { Recommends }}$}

1. the use of the "non-rotating origin" in the GCRS and that this point be designated as the Celestial Ephemeris Origin (CEO) on the equator of the Celestial Intermediate Pole (CIP),

2. the use of the "non-rotating origin" in the ITRS and this point be designated as the Terrestrial Ephemeris Origin (TEO) on the equator of the CIP,

3. that UT1 be linearly proportional to the Earth Rotation Angle defined as the angle measured along the equator of the CIP between the unit vectors directed toward the CEO and the TEO,

4. that the transformation between the International Terrestrial and Geocentric Celestial Reference Systems be specified by the position of the Celestial Intermediate Pole in the Geocentric Celestial Reference System, the position of the Celestial Intermediate Pole in the International Terrestrial Reference System, and the Earth Rotation Angle,

5. that the IERS take steps to implement this by 1 January 2003 , and

6. that the IERS will continue to provide users with data and algorithms for the conventional transformations.

Note The position of the CEO can be computed from the IAU 2000A model for precession and nutation of the CIP and from the current values of the offset of the CIP from the pole of the ICRF at J2000.0 using the development provided by Capitaine et al. (2000). The position of the TEO is only slightly dependent on polar motion and can be extrapolated as done by Capitaine et al. (2000) using the IERS data. The linear relationship between the Earth's rotation angle $\theta$ and UT1 should ensure the continuity in phase and rate of UT1 with the value obtained by the conventional relationship between GMST and UT1. This is accomplished by the following relationship:

$\theta(U T 1)=2 \pi(0.7790572732640+1.00273781191135448 \times($ Julian UT1 date -2451545.0$))$

\section{Resolution 8. Re-definition of Terrestrial Time TT}

IAU Colloquium 180 "Towards Models and Constants for Sub-Microarcsecond Astrometry"

Considering

1. that IAU Resolution A4 (1991) has defined Terrestrial Time (TT) in its Recommendation 4, 
2. that the intricacy and temporal changes inherent to the definition and realization of the geoid are a source of uncertainty in the definition and realization of $\mathrm{TT}$,

3. that this may become in the near future the dominant source of uncertainty in realizing TT from atomic clocks,

Recommends that TT be a time scale differing from TCG by a constant rate: $d T T / d T C G=1-L_{G}$, where $L_{G}=6.969290134 \times 10^{-10}$ is a defining constant.

\section{$\underline{\text { Notes }}$}

1. $L_{G}$ has been computed as $U_{G} / c^{2}$ where $U_{G}$ is the current best estimate of the geopotential at the geoid $\left(U_{G}=62636856 \mathrm{~m}^{2} \mathrm{~s}^{-2}\right)$ provided by the International Association of Geodesy Special Commission 3 in its 1999 report.

2. $L_{G}$ was introduced by the IAU Resolution A4 (1991) in its Recommendation 4. It is now used as a defining constant.

\section{Resolution 10. Coordinated Universal Time}

IAU Colloquium 180 "Towards Models and Constants for Sub-Microarcsecond Astrometry"

$\underline{\text { Recognizing }}$

1. that the definition of Coordinated Universal Time (UTC) relies on the astronomical observation of the UT1 time scale in order to introduce leap seconds,

2. the impact of unpredictable leap seconds on modern communication and navigation systems,

3. that astronomical observations provide an accurate estimate of the secular deceleration of the Earths rate of rotation

\section{$\underline{\text { Recommends }}$}

1. that the International Astronomical Union establish a working group reporting to IAU Division I at the IAU General Assembly in 2003 to consider the redefinition of UTC,

2. that this study discuss whether there is a requirement for leap seconds, the possibility of inserting leap seconds at pre-determined intervals, and the tolerance limits for UT1-UTC, and 
3. that this study be undertaken in cooperation with the appropriate groups of the International Union of Radio Science (URSI), the International Telecommunications Union (ITU-R), the International Bureau for Weights and Measures (BIPM), and relevant navigational agencies.

\section{Resolution 11. Definition of a Spectroscopic "Radial-velocity Mea- sure"}

IAU Colloquium 180 "Towards Models and Constants for Sub-Microarcsecond Astrometry"

\section{Recognizing}

1. that recently improved techniques for determining radial velocities in stars and other objects, reaching and exceeding precision levels of meters per second, require the definition of "radial velocity" to be examined;

2. that, due to relativistic effects, measurements being made inside gravitational fields, and alternative choices of coordinate frames, the concept of radial velocity being equal to the time derivative of distance, becomes ambiguous at accuracy levels around $100 \mathrm{~m} / \mathrm{s}$; and

\section{Considering}

1. that, although many effects may influence the precise shifts of spectroscopic wavelengths and frequencies, only local ones (i.e. arising within the solar system) can be reliably calculated. These local effects depend on the gravitational potential of the observer and the observer's position and motion relative to the solar system barycenter;

2. that, although the wavelength displacement (or frequency shift) corrected for such local effects can thus be derived from spectroscopic measurements, the resulting quantity cannot unambiguously be interpreted as a radial motion of the object;

Recommends that, whenever radial velocities are considered to a high accuracy, the spectroscopic result from a measurement of shifts in wavelength or frequency is given as the [barycentric] "radial-velocity measure" $c z$, after correcting for gravitational effects caused by solar-system objects, and effects by the observer's displacement and motion relative to the solar-system barycenter.

Note Here, $c$ equals the conventional speed of light $=299,792.458 \mathrm{~km} / \mathrm{s}$, and $z=$ $\left(\lambda-\lambda_{0}\right) / \lambda_{0}$, where $\lambda_{0}$ is the rest-frame wavelength and $\lambda$ the wavelength observed by a hypothetical observer at zero gravitational potential, located at, and being at rest with respect to, the solar system barycenter. The radial-velocity measure $c z$ is expressed in velocity units: to first order in $z$ it coincides with the classical 
concept of "radial velocity", while avoiding the implicit interpretation as physical motion. The solar system barycenter is defined by Resolution A4 adopted at the IAU XXIst General Assembly in 1991, and supplemented by Resolution B6 at the IAU XXIIIrd General Assembly in 1997. 